Review

\title{
Technology Adoption in Indian National Education Policy 2020: An Analysis of Pedagogical, Institutional and Human Aspects
}

\author{
1*Arnab Kundu and ${ }^{2}$ Tripti Bej \\ ${ }^{1}$ Department of Education, Bankura University, Bankura, India \\ ${ }^{2}$ Department of Science Education, Srima Balika Vidyalaya, Paschim Medinipur, India
}

\author{
Article history \\ Received: 28-06-2021 \\ Revised: 06-07-2021 \\ Accepted: 10-07-2021 \\ Corresponding Author: \\ Arnab Kundu \\ Department of Education, \\ Bankura University, India \\ Email: arnabkundu5@gmail.com
}

\begin{abstract}
The pandemic arrival of COVID-19 has brought Educational Technology (ET) especially Information and Communication Technology (ICT) into the limelight among academicians, students and policymakers. Against this backdrop, it will be pertinent to critically analyze the provisions for ET adoption in Indian National Education Policy (NEP) 2020 to add to its sharpness to give vision to Indian education for the upcoming few decades. In this rational content analysis three basic aspects of NEP 2020 pedagogical, institutional, the human factor - have been decoded, the significance of these factors has been established in existing literature and their alignment in the Policy has been evaluated. The analysis finds this vision document as successful in capturing a comprehensive ethos of technology adoption with few limitations. All three factors have been attended, moreover, the advanced mindset that the policy exhibits with greater emphasis on the dematerialization and digitalization of content are hitherto a remarkable feat in the conservative Indian educational system. Few concerns have been raised relating to the implementation of this policy, especially relating to autonomy and self-reliance at the institutional level. Besides concerns regarding issues of human factors like developing skills among teachers, collaboration, developing group mindset and efficacy have also been evolved. This analysis is well-intentioned from the point of view of policy review, restructure and renovation of any country especially the developing ones. The recommendations authors bring for institutional strengthening in a time-bound manner by forming the Working School Governing Bodies (WSGBs) and practicing the 3-E Model that may open up a new vista of ET integration at the school level.
\end{abstract}

Keywords: NEP 2020, Educational Technology, Pedagogy, Institution, Human Factors

\section{Introduction}

\section{Espoused Necessity of Technology Adoption in Education}

Man has been using technology to make their lives easy since the days unknown and his ambition has been to make technology complex to more complex. Today's cutting-edge technology has been serving human life in every sphere and education is not an exception. We have reached at the tipping points of computing and 'ephemeralization' (Fuller, 1938), a term coined by R. Buckminster Fuller in 1938 is at its highest level. The philosophy of doing everything with nothing has been taking our mind to the roots. In such a backroom the future education is going to have a massive transformation where technology will play a more active role that what it has been playing now.

The problem-based learning that is ability of learners to solve real world problems, immersion that is real time decision making and simulation that is a safe environment for problem solving going to take driving principles pf future education as presented in the Venn diagram (Fig. 1). In all three aspects technology, rather ephemeral technology will serve a major role by transforming education from a pure knowledge transfer process to the using of institutional times in solving problems using and manipulating smarter arrangements for knowledge transfer using technology like simulation of human minds through neural networks, deep learning and artificial intelligence. Everywhere ICTs are very predominant. 


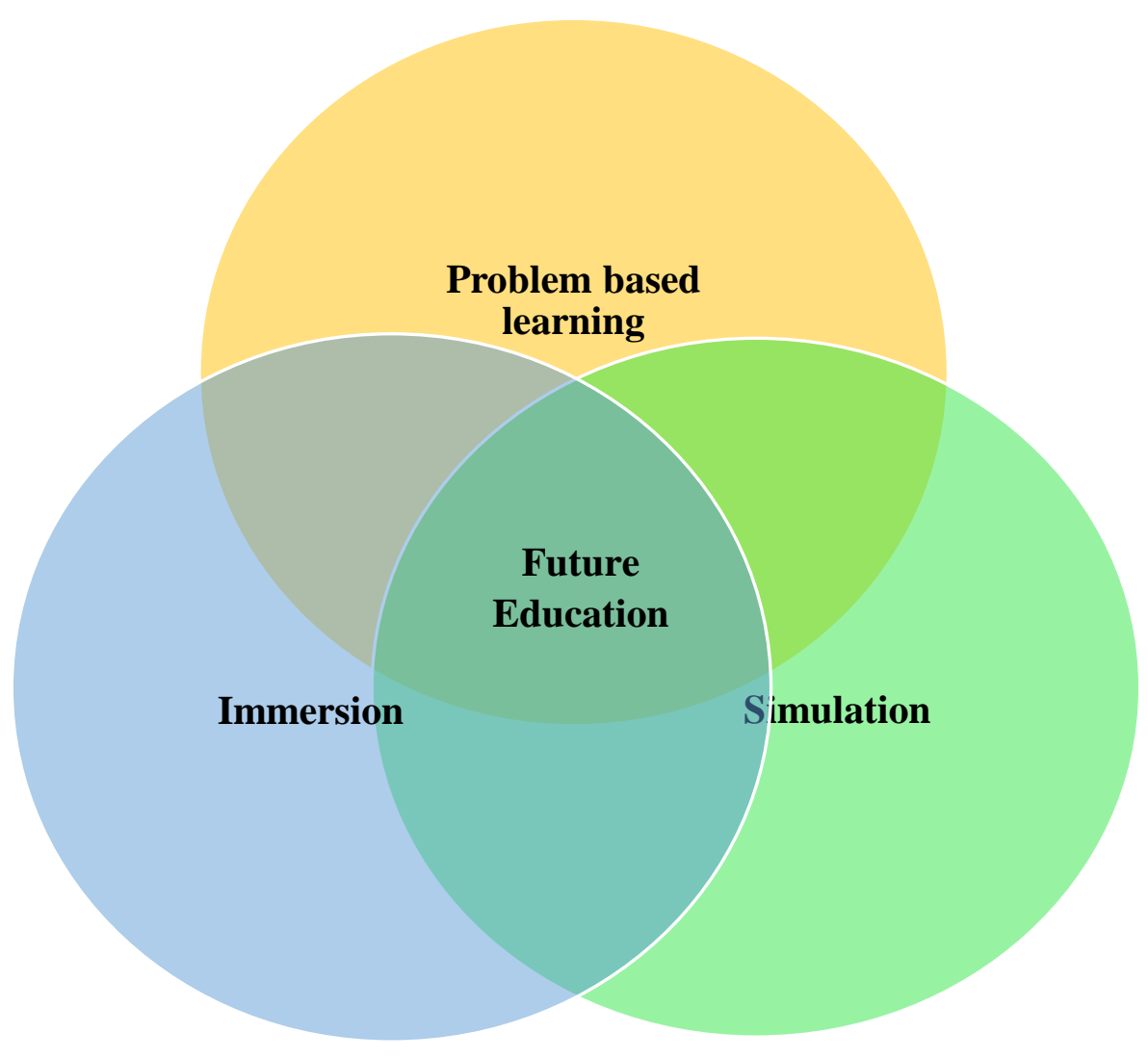

Fig. 1: Venn diagram capturing future education

The benefits of technology have been advocated in numerous studies reporting that technology will enable students' self-learning, interactive learning, continuous refinement of their learning and thus fostering their creativity (Bransford et al., 1999). Technology adoption in education is no more a novel concept. Following the examples of the developed Western Nations, India has also started the process of this adoption way back in 1992 in the Revised National Policy of Education-1992 along with several subsequent schemes and measures to utilize this potential tool of educational delivery. But the intensity and cordiality of this adoption was somewhat sloth owing to several factors starting from infrastructural limitations to the orthodox teacher-cantered pedagogic culture (Kundu and Dey, 2018). Educational technology has also been going through constant change since the time of Sidney L. Pressey's Mechanical Teaching Machines in the 1920' and now the boom in the Information and Communication Technology (ICT) with Artificial Intelligence (AI) has almost grabbed the whole attention of education technology. The pandemic outbreak of COVID-19 has undoubtedly established an unrivalled hegemony of ICT in the domain of educational technology (Kundu and Bej, 2021a). But technology use and its integration are two different things. Integration is a lot more complex phenomenon comprised of several factors like tools, techniques, skills, culture and other resources (Kundu and Bej, 2021b). Most teachers believe in the benefits of ET but feel helpless in the question of using them during pedagogy. This notion finds beautiful theoretical labelling in the book of Argyris et al. (1985) entitled 'Action Science' where they have made a distinction between 'Espoused Theory' and 'Theory-in-Use' where they asserted 'Espoused theories' are those that individual claims to follow while 'Theories-in-Use' are those than can be inferred from practical action" (p. 82). This discussion has a vital significance here, in this analysis, since teachers, an important aspect of any educational transformation, often found to have espoused or believe in the potential of educational technology inspired by constructivist learning, student-centered, outcomes-based approach and in deeplearning but whenever comes to make these theories happen in practice they follow the opposite, surface-learning, teacher-centered pedagogy, content-based approach. Phillips (2005) has made a graphical presentation of espoused theoretical characteristics of ET that needs a mention here to further illustrate the benefits of ET (Fig. 2).

\section{Technology Adoption is a Holistic Effort}

It is evident from the above diagram (Fig. 1) that ET is not leading directly to the learning, rather it has been playing the role of a tool to assist learning. Learning is an 
internal, cognitive activity which is influenced by technology, environment, humans and other factors, but it is never be delivered wholly by humans or machines. Kundu (2021) finds a mismatch between espoused theories of ET/ICT and theories in use meaning the empirical evidences of how learning takes place with ET integration and institutions/teachers perceived of teaching. Aldunate and Nussbaum (2013) have rightly pointed out that there has been an increase in the availability of ICT tools in schools at the same time there is an indication that teachers are not using these as expected. Besides this cognitive or perceptual mismatch there are several factors influencing technology adoptions in education that needs a discussion here before heading towards the content analysis which are as Ertmer (1999) found more or less the same irrespective of countries or cultures. McNaught et al. (2000) in their study mentioned three basic factors influencing the adoption of ET in general and ICT in particular which is presented in the Venn diagram in Fig. 3.

This diagram shows that mere policy making is not sufficient for technology adoption and its integration. The other two factors- culture and support- are equally important. All three factors when melodiously paly their respective roles effective cadence of adoption ensues. A large part of the Policy factor includes strategic process that is long term future preparation usually take place through four phases like policy formulation, implementation management, evaluation-feedback and commensurate policy modifications. Besides suitable leadership, adequate funding, supervision and rewarding, teaching-learning models, protecting intellectual properties and ET/IT services are also required.

Of the Culture factor, a large portion is embraced by collaboration and motivation. Group works, group mindset or collective efficacy is very vital here. The personal as well as institutional motivation like staff rewards, encouragement towards innovation, etc. matters a lot in this factor encompassing both cultural mooring of the institution and the society.

The Support factor includes a series of institutional infrastructure like ICT equipment, network, electricity, labs, etc. and encouraging management to help and facilitate this technology adoption process. Finally, when all these three factors operate in sound coordination the ideal technology adoption happen as found in the center of the diagram (Fig. 3). The implication is that mere policy is not enough unless and until it gets the support of other two factors.

As already mentioned, technology use and its proper integration are two different things hence, in most cases it was found that technology resources are lying stacked in the labs or store-rooms unused (Kundu, 2021). Thus, technology integration is a daunting task since evaluation of a tech-tool that flawlessly integrates with the predetermined curriculum need to be reasonable and being potent to elevate learners' experience without making them tiresome or feeling irrelevant (Earle, 2002). It demands change in the teacher's role (Kundu, 2018) at the same time corresponding changes in the institutional and societal roles is also inclusive (Chigona, 2015).

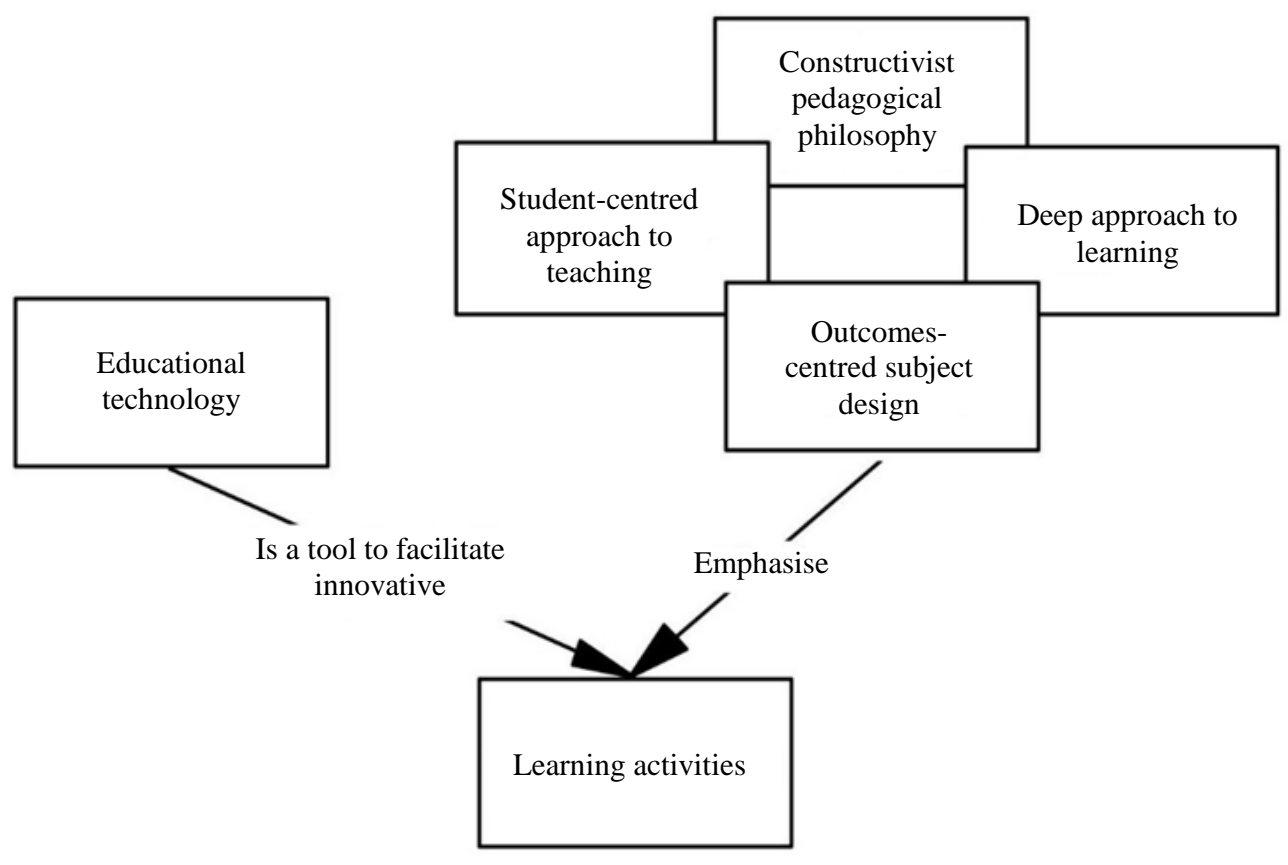

Fig. 2: Role of ET in facilitating learning activities (Phillips, 2005) 


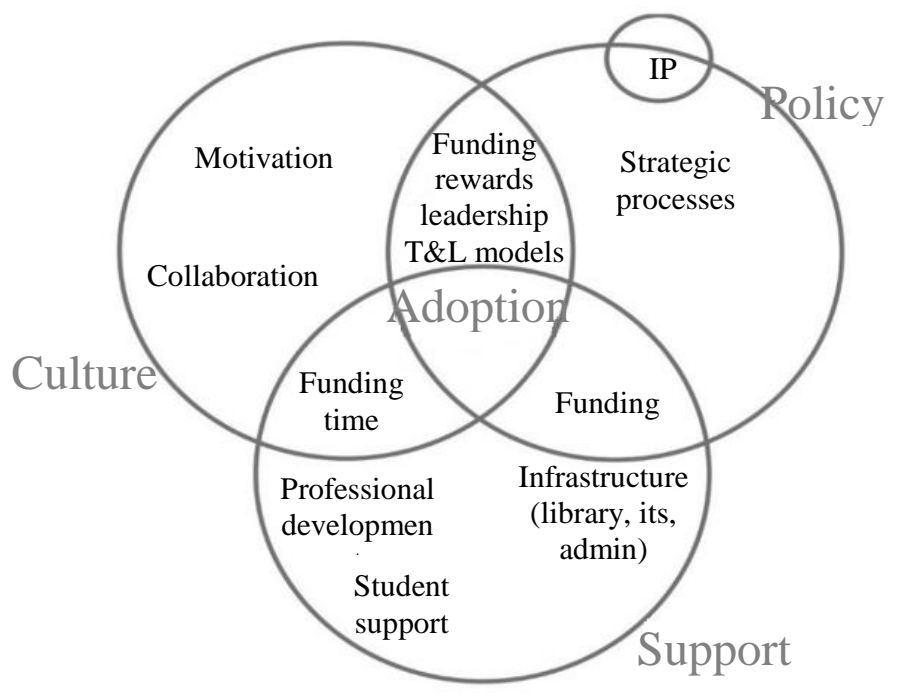

Fig. 3: Key factors behind technology adoption (McNaught et al., 2000)

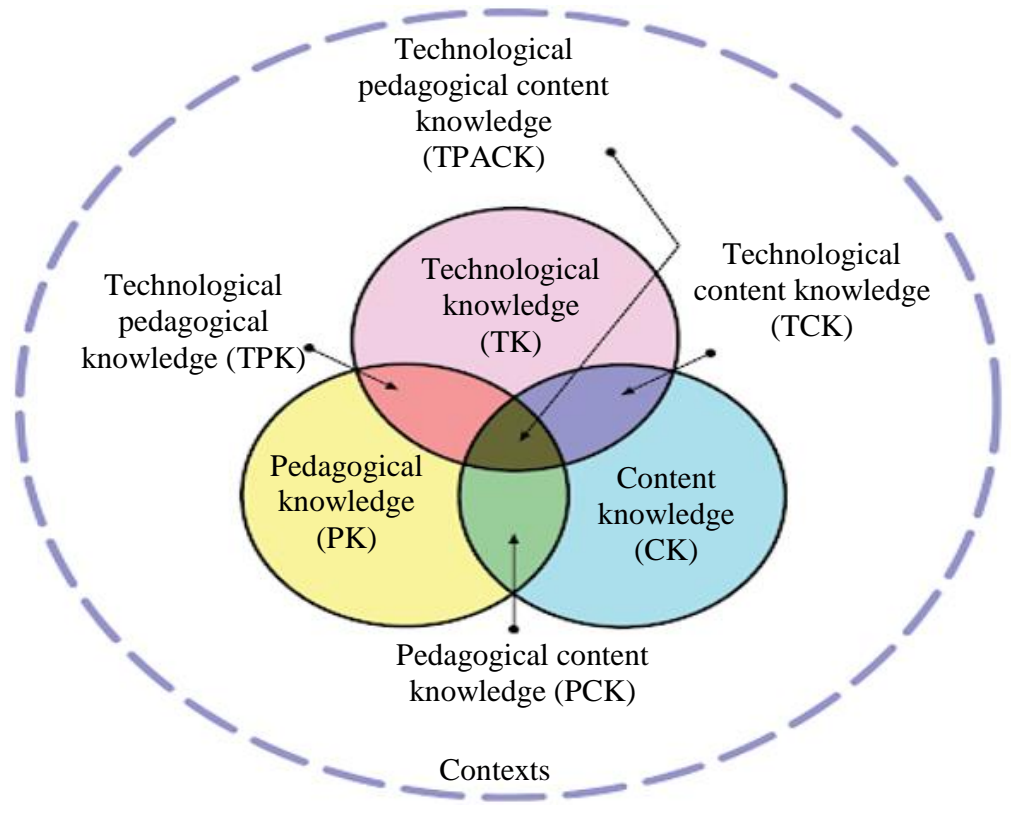

Fig. 4: TPACK Framework (Koehler and Mishra, 2008)

A short discussion on the TPACK framework developed by Koehler and Mishra (2008) could be helpful here to understand the type of skills teachers needed for a harmonious adoption of technology in education. TPACK is one of the very popular models convincingly illustrates the different kind of knowledge that a teacher needs for an effective pedagogical practice with the integration ET (Fig. 4). This will help to assess the policy content if there are provisions multifaceted human skills that teachers need to effectively adopt technology.

It talked about three main domains of skills relating to - technology, pedagogy and content- necessary for effective technology adoption and when a sound combination of these three skills happens effective integration of ET take place, as shown in the centre of this Venn diagram. It asserts that technology knowledge must be supported with content and pedagogical knowledge to give students a wholistic learning experience. TPACK model also important in developing positive culture among teachers where they can discuss about their experiences with each-other regarding trials and errors during their classroom integration experiences (Adams, 2019). The main drawback in this espoused theoretical supposition, as Ertmer (1999) and Kundu and Bej (2020b) rightly pointed out, is the extreme work pressure of timely completion of curriculum on the teachers that they hardly 
find time to think about themselves, about their skills, convictions, capacities and to update them with new thoughts, methods and technologies.

\section{Human Factors in Technology Adoption}

Another important aspect of technology adoption is the human factors. Teachers are there at the centre of any educational transformation hence, their perceptions, attitude, behavioural intension, mindset, efficacy, self-concept matters in the case of ET adoption (Guoyuan et al., 2010; Chigona, 2015; Kundu, 2020). This proposition has been effectively presented in the Technology Acceptance Model (TAM) (Fig. 5) originally developed by Fred D. Davis (1989) and its subsequent modifications.

TAM shows human factors are vital in ET adoption and without sound coordination of these human factors like attitude, intention, perceived usefulness, perceived ease of use, etc. this adoption will not be effective or may kiss a failure. Hence, human factor has been adopted as an important aspect in this analysis of the content.

\section{Brief Detailing of NEP 2020}

The much waited and anticipated National Education Policy (NEP) 2020 was formally released by the Government of India on $29^{\text {th }}$ July 2020 with the proclaimed moto 'Educate Encourage Enlighten'. This policy document comes out after 34 years and is remarkably different in several facets from its predecessor the National Policy of Education 1986 having much more focus of the infusion of $21^{\text {st }}$ century skills among students, renewed interest on the research and innovations, with the ultimate goal of making India a knowledge society (Nandini, 2020).

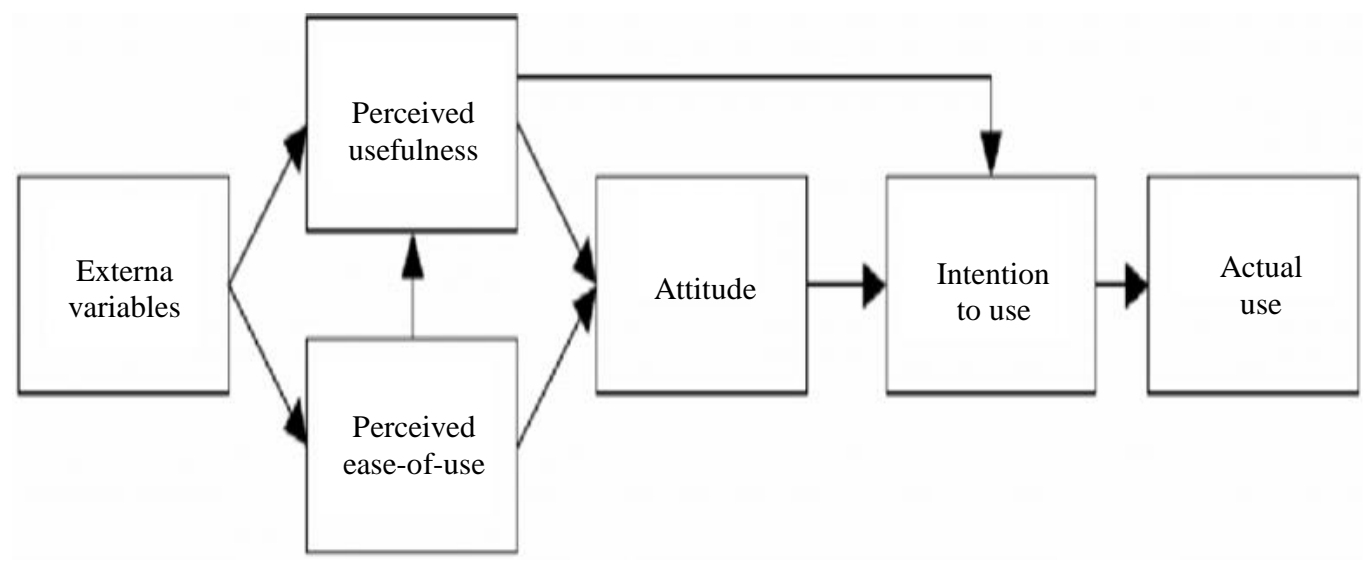

Fig. 4: Technology Acceptance Model (in Davis, 1989)

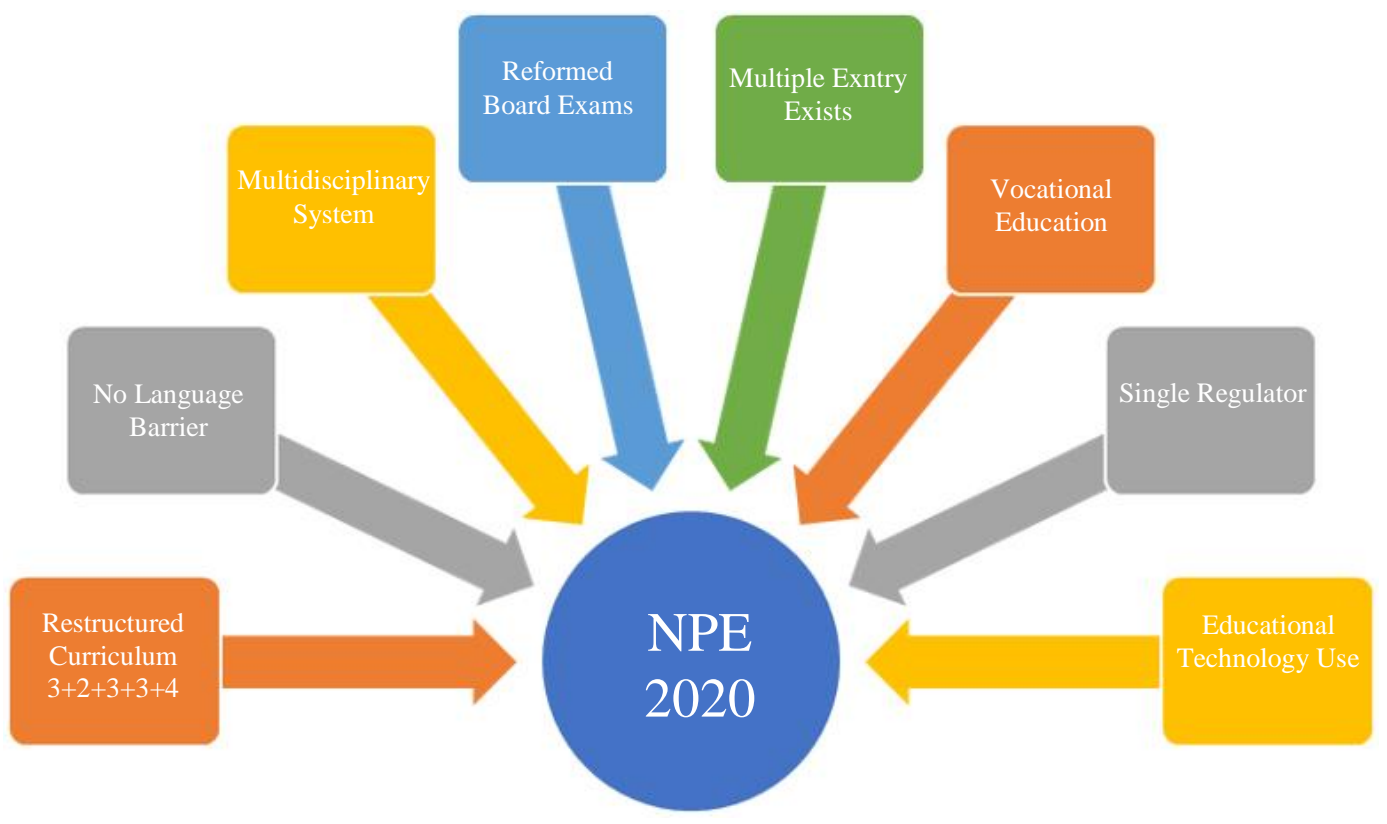

Fig. 6: Illuminating shifts in NEP-2020 
It has been containing within it the lineage of the previous objectives as well like proclaimed agenda for UNESCO Sustainable Development under (SDG4) endorsed by India earlier seeks to "ensure inclusive and equitable quality education and promote lifelong learning opportunities for all by 2030" (NEP, 2020). The whole policy document (NEP2020 ) is comprised of 66 pages. Going through the whole vision document the following illuminating shifts from earlier Policies have come out as presented in Fig. 6.

\section{Methodology}

\section{Research Objectives}

Considering the importance of the three aspectspedagogical, institutional, human- in ET adoption and integration, this study took up these vital factors to assess and assert their place in the NEP 2020. Pursuing this aim three research questions were set as follows:

RQ1: What provisions are there to develop technologyenabled pedagogy?
RQ2: What necessities stressed on to strengthen institutional ET adoption?

RQ3: What scopes recommended for upskilling human resources?

\section{Research Design}

This study was basically a Conceptual Content Analysis of NEP 2020, ranging from Pages 56-60. Content analysis is basically determination of certain or contents within some chosen qualitative data. Hsieh and Shannon (2005) said content analysis is a research tool used to determine the presence of certain words, themes, or concepts within some given qualitative data (i.e., the chosen text for analysis). Elo et al. (2014) divided this method into two groups-conceptual analysis and relational analysis. By conceptual analysis they mean the existence and frequency of certain concepts in a text while relational analysis is a step ahead of it that examine the relationships among chosen concepts in a text.

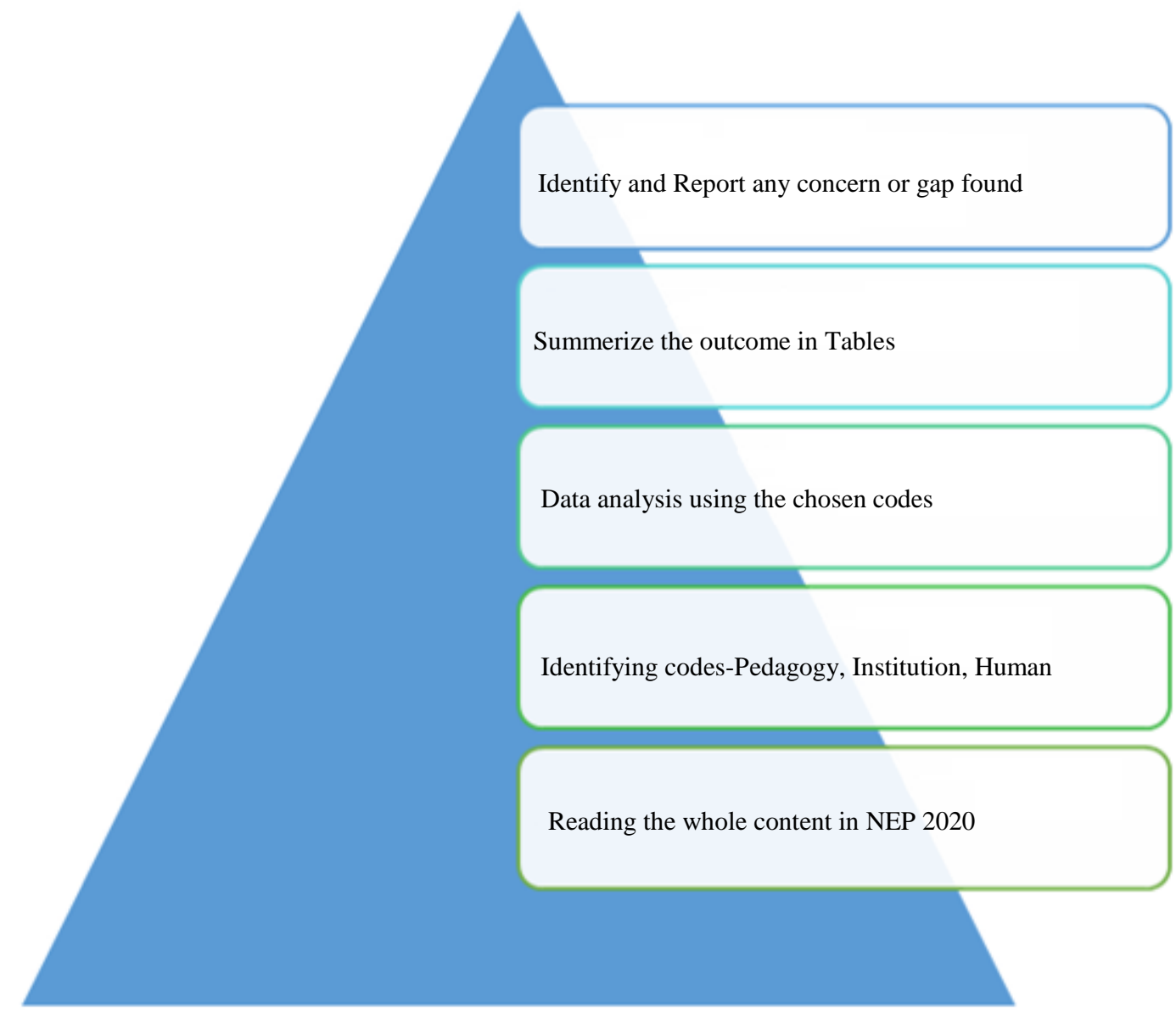

Fig. 7: Steps followed in content analysis 
Typically, conceptual analysis is generally thought of content analysis. In conceptual analysis, a concept is chosen for examination and the analysis involves quantifying and counting its presence. The main objective is to inspect the occurrence of selected terms in the data. Terms may be explicit or implicit. Explicit terms are easy to identify. Coding of implicit terms is more complicated: You need to decide the level of implication and base judgments on subjectivity (issue for reliability and validity). Therefore, coding of implicit terms involves using a dictionary or contextual translation rules or both. In this study the chosen content has been analysed following the steps stated below in Fig. 7.

\section{Results}

\section{Findings on Main provisions for ET adoption}

After a deep reading through the policy content the main ET adoption provisions that are evolved have been presented in Fig. 8. The main thrust areas that have been chosen for the use of ET are teaching-learning and assessment, teachers' professional development, enhancing educational opportunities to the all especially to the students with special needs (SSN), streamlining of educational management and administration and AI, Virtual reality, Robotics, Simulation and other "disruptive technologies that are expected to change the way we live and, therefore, change the way we educate students" (58). Another important aspect is the proposal to establish National Educational Technology Forum (NETF), an autonomous body to discuss, strategize and monitor technology led education at all level -school to university. E-courses will be developed under NEFT in eight regional languages initially (IE, 2020) and apposite virtual labs will be developed which will help spreading e-learning at every nook and corner of this country provided having suitable digital infrastructure available to the stakeholders. A lot of emphasis has been put on the creation of digital infrastructure and strengthening the existing ones. The Digital India Scheme that has been initiated in 2015 to make this country digitally adroit and capable, has been entrusted with duty to enhance digital infrastructure for ET adoption like creation of online teaching platforms, virtual labs, digital repositories, eassessment tools, quality digital contents, etc. Among other developmental aspects the creation of the Digital Infrastructure for School Education (DIKSHA) e-portal is very relevant offering learning e-material suitable for school curriculum to teachers, students and parents, at the same time offering betting professional development programs for the teachers. The existing Study Webs of Active-Learning for Young Aspiring Minds (SWAYAM) e-portal designed for higher education will work hand in hand with the DIKSHA and complement each other in the future course. Assessment, Review and Analysis of Knowledge for Holistic Development (PARAKH) is an important step towards assessment reform proposed in this NEP 2020 to set up as a standard-setting national assessment centre all recognized school boards that collectively aim to transcends the working procedures of the school boards towards holistic assessment from existing high-stakes paper-pen examinations. This is a novel forward step keeping a high object in front. The policy document has also identified the major setbacks towards this ET adoption that includes bridging the current deep digital divide across the country, poor digital competence among teachers, unavailability of open and public digital resources, low mobile and internet penetration in the rural areas, shortage of eresources in institutions and limitation of teaching certain subjects online.

\section{Thematic analysis of the Findings}

The results of the above analysis have been put and tested through the touchstone of the selected three thematic aspects evolved from the existing literature as discussed earlier and the findings are reported in the following diagram in Fig. 9. Findings relating to developing pedagogical aspects of the ET adoption are mainly centered around DIKSHA \& SWAYAM entrusted to enhance pedagogical capabilities of teachers and NEFT to innovate new strategies and methods of technology integration. But limitations are there regarding school level practices of ET integrated pedagogy. Pedagogy tends to be less effective until it is made phenomenologically reflective. Hence, the in-school trainings have wider implications in enhancing teaching efficacy and efficiency. Institutional strengthening is mainly concerned with equipped the institutions with adequate e-learning contents and digital devices and this duty has been entrusted with the Digital India Scheme. Here also one limitation evolved regarding the acute shortage of e-devices among millions of students that has been very evident during this COVID-19 pandemic time (Kundu and Bej, 2020b), but no solid guideline evolved to equip students with mobile or e-devices. The major concern has been evolved in strengthening the human-elements. Most of the provisions that come out regarding these aspects are mostly theoretical and subjecting to future orientation. But no rational direct and immediate plan of action has been advocated for strengthening the digital capacity, efficiency and efficacy of the teachers and students. The provisions for teachers training prescribed are found too superficial and far from, practical implementation which may befall the bins of future unused teaching polices. Until and unless school level strong polices have been taken to strengthen teachers' usability of ET the efficient generation of teaching staff will not be flourished who could efficiently develop e-contents and effectively implement them in their teaching. 


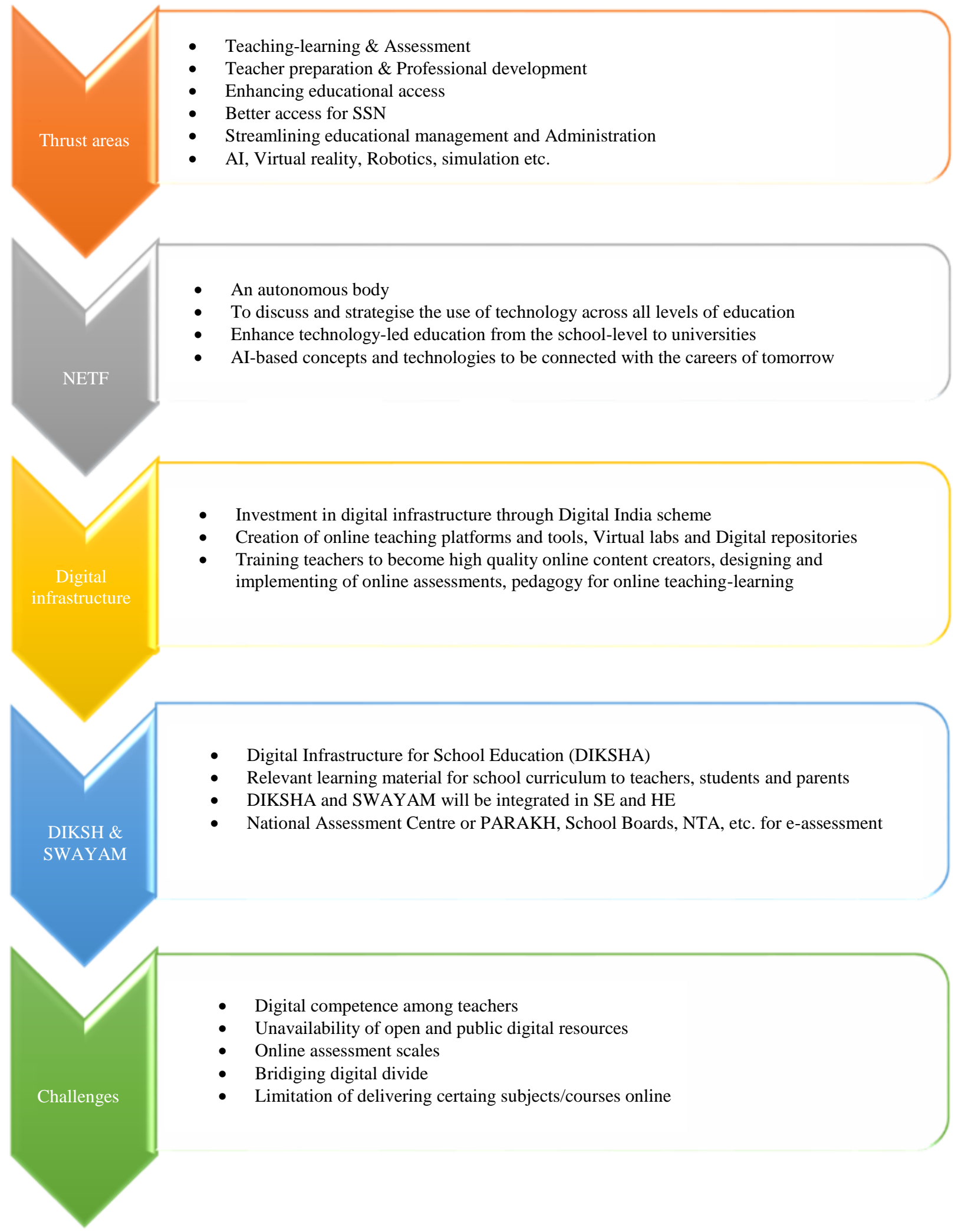

Fig. 8: Main provisions for ET adoption in NEP 2020 


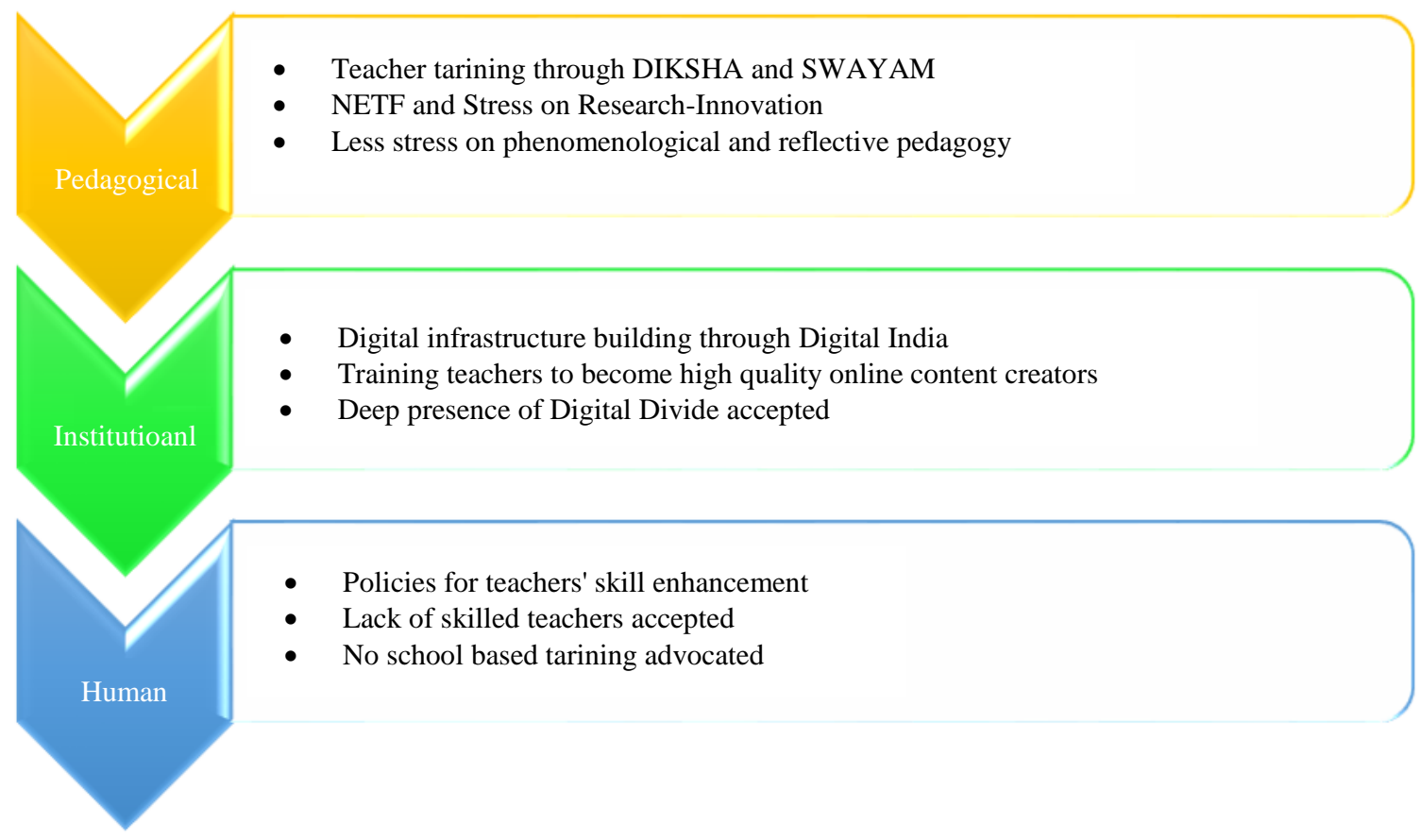

Fig. 9: Thematic analysis of the ET adoption provisions

\section{Discussion}

\section{Discussion on RQ1}

Regarding the first research question "what provisions are there to develop technology-enabled pedagogy" it came out that several provisions have been made to develop technology-enabled pedagogy, especially through DIKSHA \& SWAYAM e-portals. The creation of the DIKSHA portal is something new and optimistic which is supposed to provide "teaching-learning $e$ contents developed by all States in all regional languages, as well as by the NCERT, CIET, CBSE, NIOS and other bodies/institutions and will be uploaded onto the DIKSHA platform. This platform may also be utilized for Teacher's Professional Development through e-content" (p.57). National Educational Technology Forum (NETF) is another important step that has been entrusted with the duty to conduct research and development of technopedagogy and communicate with teachers through discussion forums. It is claimed that NETF "will create a platform for the free exchange of ideas on the use of technology to enhance learning, assessment, planning, administration and so on, both for school and higher education" (NEP. 2020). The provision is also there that NEFT will work to connect "knowledge and practice, organizing multiple regional and national conferences, workshops, etc. to solicit inputs from national and international educational technology researchers, entrepreneurs and practitioners" (p.57). These steps to enhance and equip teachers with suitable pedagogy will certainly be fruitful and effective as already evolved in the literature that has been presented in the TPACK framework. But all these are theoretical propositions only be effective with a strong implementation plan where the policy was found to offer a narrow passage. There are few scopes left for school-based ET adoption, practice and proliferation. Besides no attempts have been proposed to develop e-contents at the school level because it the schools which are better equipped to prepare pedagogy for their students considering their needs and capacities (Kundu and Bej, 2021b). This kind of social pedagogy could be more effective in creating a convincing technologyenabled teaching-learning culture (Schoone, 2020) "where a seamless exchange between what is taught and the lived experiences of students was created" (p.5).

\section{Discussion on $R Q 2$}

The second research theme was "what provisions are there to strengthen institutions for ET adoption" and the findings revealed that the policy document itself unequivocally accepts the deep digital divide and acute shortage of e-equipment in schools present across the country making inequality in the access of ET. Hence the point is given much attention and arrangements since "the benefits of online/digital education cannot be leveraged unless the digital divide is eliminated through concerted efforts, such as the Digital India campaign and the availability of affordable computing devices. It is important that the use of technology for online and digital education adequately addresses concerns of 
equity" (p.58). The supporting literature also claims that infrastructural support is a vital parameter of ET adoption (McNaught et al., 2000). The policy documents focus on this point and proposed that the digital equipment pool of the institutions will be strengthened in a phased manner through the Digital India campaign. The lack of skilled teachers will also be attenuated through their training using e-portals like DIKSHA or SWAYAM as it said "teachers will undergo rigorous training in learnercentric pedagogy and on how to become high-quality online content creators themselves using online teaching platforms and tools" (p.59). There have been many discussions on the development of e-contents and towards a gradual shift from materialization to digitization. To quote the policy document it is said that " $a$ digital repository of content including the creation of coursework, Learning Games \& Simulations, Augmented Reality and Virtual Reality will be developed, with a clear public system for ratings by users on effectiveness and quality" (p.59). But most of these propositions are futureoriented and chances to fail with the change of government and its focus areas. Besides it depends on the integrity and intensity of intention among the implementation authority that school enhances their digital resource pool. Here also a school-based approach would be beneficially entrusted with the duty to empower their respective stakeholders digitally with a strong supervision mechanism.

\section{Discussion on RQ3}

It revealed that human-technology interaction is a vital facet of ET adoption as the TAM discussed. Hence the third research question "what scopes are there for upskilling human resources" centered around this notion and went out to investigate this issue. The finding showed the policy document accepted the lack of skilled human force in ET adoption and focused on their reskilling and upskilling. These efforts don't appear sufficient to touch the wide variety of demands and psycho-social variations among teachers and students. They are varying so far as their attitude, awareness, belief, self-image, societal upbringings are concerned. Professor Marmar Mukhopadhyay in the Preface of his book Total Quality Management in Education has righty asserted that educational quality is far different from the quality of industrial products since humans are complex body-mindspirit organisms and not just knowledge and skill configuration (Mukhopadhyay, 2020). Human development implies comprehensive development of the potentials already in them. Here questions come how far the centrally inflicted schemes would effective to upskill human elements according to their individuality. Besides, poor provision has been evolved in this analysis concerning decentralization and school-based teachers' capacity building keeping in front of their needs. But the policy documents seem aware of this limitation hence left a place for further ramification of their visions as it appears in the lines "since technology is rapidly evolving and needs specialists to deliver high-quality e-learning, $a$ vibrant ecosystem has to be encouraged to create solutions that not only solve India's challenges of scale, diversity, equity, but also evolve in keeping with the rapid changes in technology, whose half-life reduces with each passing year" (p.60).

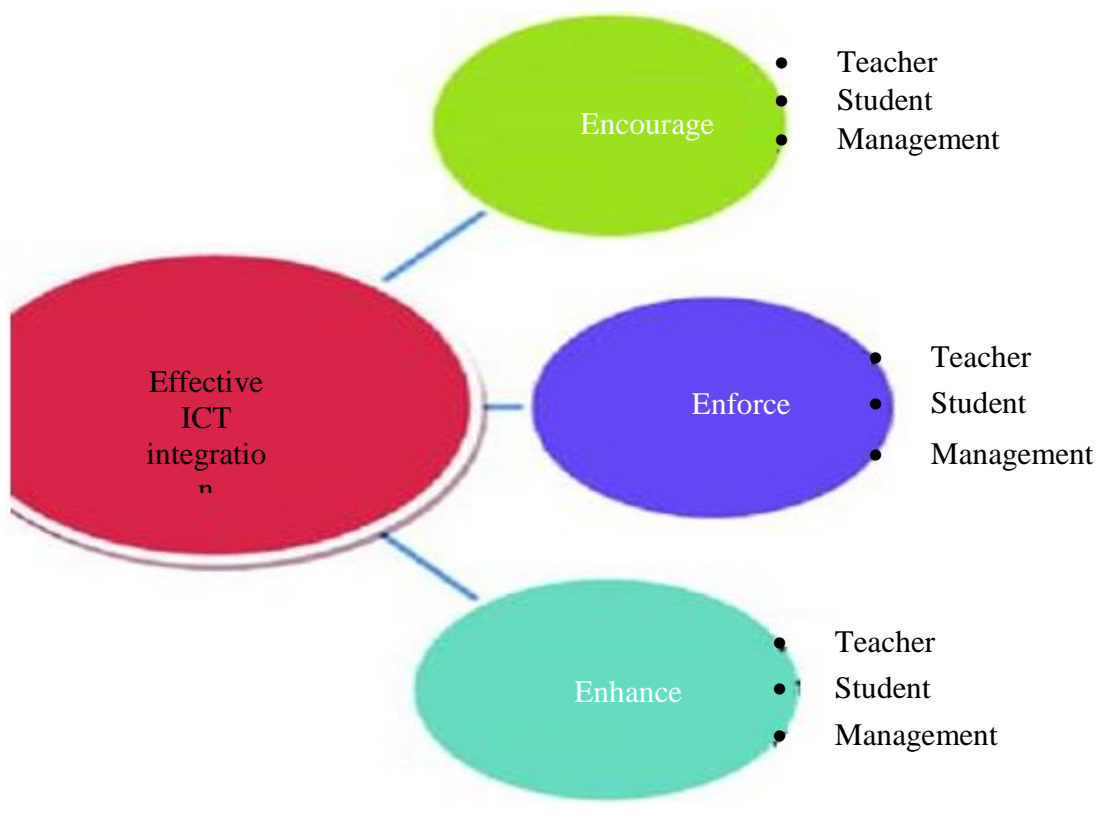

Fig. 10: 3E-Model for ET integration in pedagogy (Kundu and Bej, 2020a) 
These human elements are under the direct control of the concerned institutions hence, proper institution planning could help to enhance efficacy, efficiency and empowerment. In this respect sound institutional planning could cordially address this issue relating to the ET integration and here the 3E-Model (in Fig. 10) along with the program of action for its implementation as stated in Kundu and Bej (2020) may be tested under the overall monitoring of the Working School Governing Bodies (WSGBs). To quote Professor Mukhopadhyay mere imitating Western wisdom will not hold water in our Indian context rather local culture and local ethos needs more attention to secure quality education (Mukhopadhyay, 2020).

\section{Conclusion}

It is worth mentioning John Dewey's famous remark, "If we teach today's students as we taught yesterday's, we rob them of tomorrow." This advocacy for constant change still resonates across today's educational climate irrespective of geographical boundaries. Responding to this forward-thinking notion Prof. Mukhopadhyay, a renowned figure in planning and administration of educational technology in India in an Editorial of ETMA (2021) has beaconed this change saying that teachers must experience learning before asking students to learn in a particular way. This notion of constant change in pedagogy has rightly been captured in the NEP 2020. It has been a much more forwards move in comparison to its predecessor the National Policy of Education NPE (1986) and has rightly overcome its earlier hesitant positioning with the technology's disruptive implications for education. Respecting the 21 st Century skills, demands of the tech-savvy generation and the potential of ET, it has rightly been emphasized for securing e-resources, digital infrastructure, developing digital skills and promoting the role of ET in the improvement of educational processes and outcomes. It desires delegation of authority, ponders over the cooperation between the various industry stakeholders and educational institutions and active role of school management committees also. There is the provision for NETF to adopt and ingest industry-led best practices for the institutions through constant research and innovations.

But the success of any policy depends on its means and methods of implementation wherefrom few concerns evolved in course of this analysis regarding digital divide, institutional participation and upskilling human elements. The study finds that the focus on bridging the prevalent deep digital divide across this huge country is not sufficient and time-bound when as per a recent survey conducted in January 2021 internet penetration in India stood at $45.0 \%, 79.0 \%$ of the total population use mobile and $4.4 \%$ household has a computer but this statistics cuts poor streamlining the rural areas (Datareportal, 2021). Digital infrastructure is a prerequisite for technology-integrated education hence speedy time-bound redressal of this digital divide is indispensable. This has been proved during the COVID-driven virtual education scheme making educational access for the majority of Indian students almost impossible (Kundu and Bej, 2020a), even this is the case in most of the developing nations across the globe as well and this impact is more potent among the marginal socioeconomic households who were significantly disadvantaged (Kundu and Bej, 2021). The success of any policy is dependent on the flourishment of suitable culture and support system as already discussed in the literature. For both these factors, institutional empowerment is necessary. The institutions need to self-reliant over teacher training, eresources, or e-content preparation and delivery. But the analysis reveals a lack of adequate attention to this major aspect of ET adoption. Considering this loophole this study wants to recommend the creation of a separate policy implementation body like the Working School Governing Bodies (WSGBs) in each school or with a group of schools that would look after the issues relating to the promotion of ET integrated pedagogies in concerned schools. Human elements associated with education cannot be ignored and TAM has shown users' attitude, awareness, perceived usefulness, perceived ease of use and behavioural intention affect their actual use of technology. Teachers and students are the prime human elements associated with ET adoption hence, adequate attention needs to be given to their needs, inclinations, beliefs and aspirations where the policy document appears with a narrow prospect. Moreover, this facet is directly linked with the institutional jurisdiction that falls in the domains of culture and support in the discussed model (McNaught et al., 2000) in this study. Sound school-based institutional planning could address these human aspects of ET adoption. Here also the 3E-Model along with the 'Program of Action' for its implementation as stated in Kundu and Bej (2021) may be tested to 'Encourage, Enforce and Enhance' the whole cultural ethos on the institutions under the overall monitoring of the WSGBs. Finally, a revision focussing school-based ET practices and proliferation of ET pools can bring the technology adoption as recommended in NEP 2020 to face a success. For this time bound approach to achieve these targets is also a necessary for the policy provisions to be effective.

\section{Acknowledgement}

The authors acknowledge the Policy Making Body incharge of NEP-2020, Ministry of Education, India for making such a comprehensive vision document and 
pushing the whole nation towards a new outlook. The authors acknowledge the Educational Technology and Management Academy (ETMA) and its chief architect Professor Marmar Mukhopadhyay, an eminent Indian educationist for their tireless attempt to make whole the country think on educational technology with a renewed broader outlook. The authors also express their heartfelt gratitude towards this USA based esteemed journal, its editorial team, and reviewers for acknowledging our work and allowing an access through in such a short time.

\section{Author's Contributions}

Arnab Kundu: Main idea, making framework, and revisions.

Tripti Bej: Advise and policy revision.

\section{Ethical Declarations}

In this study, all declared ethical measures were followed. No single line in this review is copied from any other sources and wherever needed quoted with due citations.

\section{References}

Adams, C. (2019). TPACK Model: The Ideal Modern Classroom. Technology and the Curriculum: Summer 2019. https://techandcurr2019.pressbooks.com/chapter/tpa ck-modern-classroom/

Aldunate, R., \& Nussbaum, M. (2013). Teacher adoption of technology. Computers in Human Behavior, 29(3), 519-524.

https://www.sciencedirect.com/science/article/abs/pi i/S074756321200297X

Argyris, A. C., Putnam, R., \& Smith, DM. (1985). Action Science: Concepts, Methods and Skills for Research and Intervention. Wiley (Jossey-Bass), California, USA. https://actiondesign.com/resources/readings/actionscience

Bransford, J., Bransford, J. D., Brown, A. L., \& Cocking, R. R. (1999). How people learn: Brain, mind, experience, and school. National Academies Press. https://www.amazon.com/How-People-Learn-

Experience-Expanded/dp/0309070368

Chigona, A. (2015). Pedagogical shift in the twenty-first century: Preparing teachers to teach with new technologies. Africa Education Review, 12(3), 478-492. https://www.tandfonline.com/doi/abs/10.1080/1814 6627.2015.1110912

Datareportal (2021). Digital 2021: India. https://datareportal.com/reports/digital-2021-india

Davis, F. D. (1989). Perceived usefulness, perceived ease of use and user acceptance of information technology. MIS quarterly, 319-340. https://www.jstor.org/stable/249008
Earle, R. S. (2002). The integration of instructional technology into public education: Promises and challenges. Educational technology, 42(1), 5-13. https://www.jstor.org/stable/44428716

Elo, S., Kääriäinen, M., Kanste, O., Pölkki, T., Utriainen, K., \& Kyngäs, H. (2014). Qualitative content analysis: A focus on trustworthiness. SAGE open, 4(1), 2158244014522633. https://journals.sagepub.com/doi/full/10.1177/2158244 014522633

Ertmer, P. A. (1999). Addressing first-and second-order barriers to change: Strategies for technology integration. Educational technology research and development, 47(4), 47-61. https://link.springer.com/article/10.1007/bf02299597

ETMA. (2021, May). Education Matters @ ETMA: 7th issue of ETMA's e-magazine. https://etmaindia.in/1383-etma-presents-7th-issueof-etmas-e-magazine-educationmattersetma

Fuller, R. Buckminster (1938). Nine Chains to the Moon, Anchor Books, pp. 252-59. https://www.rationalargumentator.com/index/blog/ta g/buckminster-fuller/

Guoyuan, S., Valcke, M., Johan, V., Tondeur, J., \& Zhu, C. (2010). Predicting ICT integration into classroom teaching in Chinese primary schools: exploring the complex interplay of teacher-related variables. Journal of Computer Assisted Learning. 27. 160-172. https://link.springer.com/article/10.1007/bf02299597

Hsieh, H. F., \& Shannon, S. E. (2005). Three approaches to qualitative content analysis. Qualitative health research, 15(9), 1277-1288. https://journals.sagepub.com/doi/abs/10.1177/10497 32305276687

IE. (2020). New Education Policy 2020 HIGHLIGHTS: All you need to know about NEP.

Koehler, M. J., \& Mishra, P. (2008). Introducing TPCK. AACTE Committee on Innovation and Technology (Ed.), The handbook of technological pedagogical content knowledge (TPCK) for educators (pp. 3-29). Mah-wah, N]: Lawrence Erlbaum Associates.

Kundu, A. \& Dey, K. N., (2018). Barriers to Utilizing ICT in Education in India with a Special Focus on Rural Areas. International Journal of Scientific Research and Reviews. 7(2). Pp.341-359, https://doi.org/10.13140/RG.2.2.14437.73449

Kundu, A. (2018). A Study on Indian Teachers' Roles and Willingness to Accept Education Technology. International Journal of Innovative Studies in Sociology and Humanities. 3(9). https://doi.org/10.13140/RG.2.2.24762.31687

Kundu, A., \& Bej, T. (2021a). COVID-19 response: students' readiness for shifting classes online. Corporate Governance: The International Journal of Business in Society. https://www.emerald.com/insight/content/doi/10.1 108/CG-09-2020-0377/full/html 
Kundu, A., \& Bej, T. (2021b). Ingestion and integration of ICTs for pedagogy in Indian private high schools. ELearning and Digital Media, 18(2), 163-184. https://journals.sagepub.com/doi/abs/10.1177/20427 53020957493

Kundu, A. (2020), "Toward a framework for strengthening participants' self-efficacy in online education", Asian Association of Open Universities Journal, $15(3)$ $\mathrm{pp}$. $351-370$ https://doi.org/10.1108/AAOUJ-06-2020-0039

Kundu, A. (2021). A Sound Framework for ICT Integration in Indian Teacher Education. International Journal of Teacher Education and Professional Development (IJTEPD), 4(1), 49-67. http://doi.org/10.4018/IJTEPD.2021010104

McNaught, C., Phillips, R., Rossiter, D., \& Winn, J. (2000). Developing a framework for a useable and useful inventory of computer-facilitated learning and support materials in Australian universities. https://researchrepository.murdoch.edu.au/id/eprint/ $12299 /$

Mukhopadhyay, M. (2020). Total Quality Management in Education (3rd Edition), New Delhi: Sage Publications. https://in.sagepub.com/en-in/sas/totalquality-management-ineducation/book274122\#preview
Nandini, ed. (2020, July 29). "New Education Policy 2020 Highlights: School and higher education to see major changes". Hindustan Times.

NPE. (1986). "National Education Policy 1986". National Informatics Centre. pp. 38-45. http://mhrd.gov.in/sites/upload_files/mhrd/files/NPE -1968.pdf

NPE. (2020). National Education Policy 2020. Ministry of Education. Government of India. New Delhi. https://www.education.gov.in/sites/upload_files/mhr d/files/NEP_Final_English_0.pdf

Phillips, Rob. (2005). Pedagogical, institutional and human factors influencing the widespread adoption of educational technology in higher education. ASCILITE. The Australasian Society for Computers in Learning in Tertiary Education. https://www.ascilite.org/conferences/brisbane05/blog s/proceedings/62_Phillips.pdf

Schoone, A. (2020). Returning to the heart of teaching: Social pedagogy as phenomenological pedagogy. International Journal of Social Pedagogy, 9(1): https://doi.org/10.14324/111.444.ijsp.2020.v9.x.013 